\title{
Cosmic dust evolution: The challenges for NIKA2
}

\author{
Frédéric Galliano ${ }^{1, *}$ and the DustPedia collaboration \\ ${ }^{1}$ AIM, CEA, CNRS, Université Paris-Saclay, Université Paris Diderot, Sorbonne Paris Cité, F-91191 \\ Gif-sur-Yvette, France
}

\begin{abstract}
I summarize a recent study aimed at constraining dust evolution timescales in nearby galaxies. We were able to quantify the efficiency of dust condensation in SN II ejecta, grain growth in the ISM and dust destruction by SN II blast waves. Around Solar metallicity, our results are consistent with what we know in the Milky Way, that is dust production is dominated by grain growth. We however show that this changes with metallicity. The dust content in very-low-metallicity systems is dominated by grains that have formed in SN II ejecta. I end this article with a discussion about the potential of NIKA2 to study dust evolution in galaxies.
\end{abstract}

\section{Introduction}

Interstellar dust is a key physical ingredient of galaxies, obscuring star formation, regulating the heating and cooling of the gas, and building-up chemical complexity. Its properties are however difficult to estimate, as there are important uncertainties about the constitution of the grain mixture in the diffuse Interstellar Medium (ISM) of the Milky Way, where dust models are calibrated. In addition, dust properties are known to drastically evolve as a function of their local physical conditions (gas density, radiation field, shocks) and as a function of the history of the galaxy (enrichment of the ISM by successive waves of star formation). It is thus crucial to quantify this evolution.

Cosmic dust evolution models attempt to account for the balance between the main dust evolution processes, in a global way ( $c f$. [1] for a review). They however rely on simple descriptions of the evolution mechanisms and depend on the value of uncertain tuning parameters, such as sticking coefficients, binding energies, sputtering yields, etc. We have attempted to provide an empirical estimate of these tuning parameters, using a sample of $\simeq 800$ nearby galaxies.

\section{Galaxy sample and SED Modeling}

In order to constrain a dust evolution model, we need to estimate the following global quantities, for a large sample of galaxies: the dust mass, $M_{\text {dust }}$, the gas mass, $M_{\text {gas }}$, the stellar mass, $M_{\star}$, the metallicity, $Z$, and the Star Formation-Rate (SFR). We have used the DustPedia sample [2]. It provides homogeneous multi-wavelength photometry [3], with ancillary $\mathrm{H}_{\mathrm{I}}$ and

\footnotetext{
*e-mail: frederic.galliano@cea.fr
} 
CO observations. The stellar mass and SFR have been derived by [4], using ultraviolet-to$\mathrm{mm}$ Spectral Energy Distribution (SED) fitting. The metallicity have been estimated with a consistent calibration by [5].

One of the limitations of DustPedia is its selection of objects larger than $10^{\prime}$, excluding most local dwarf galaxies. Yet, the latter are crucial to constrain dust evolution, as they sample the low-metallicity regime corresponding to the early stages of the dust build-up. To overcome this deficiency, we have added to our sample the sources from the Dwarf Galaxy Survey (DGS, [6-9]). In total we have $\simeq 800$ objects covering a large metallicity range.

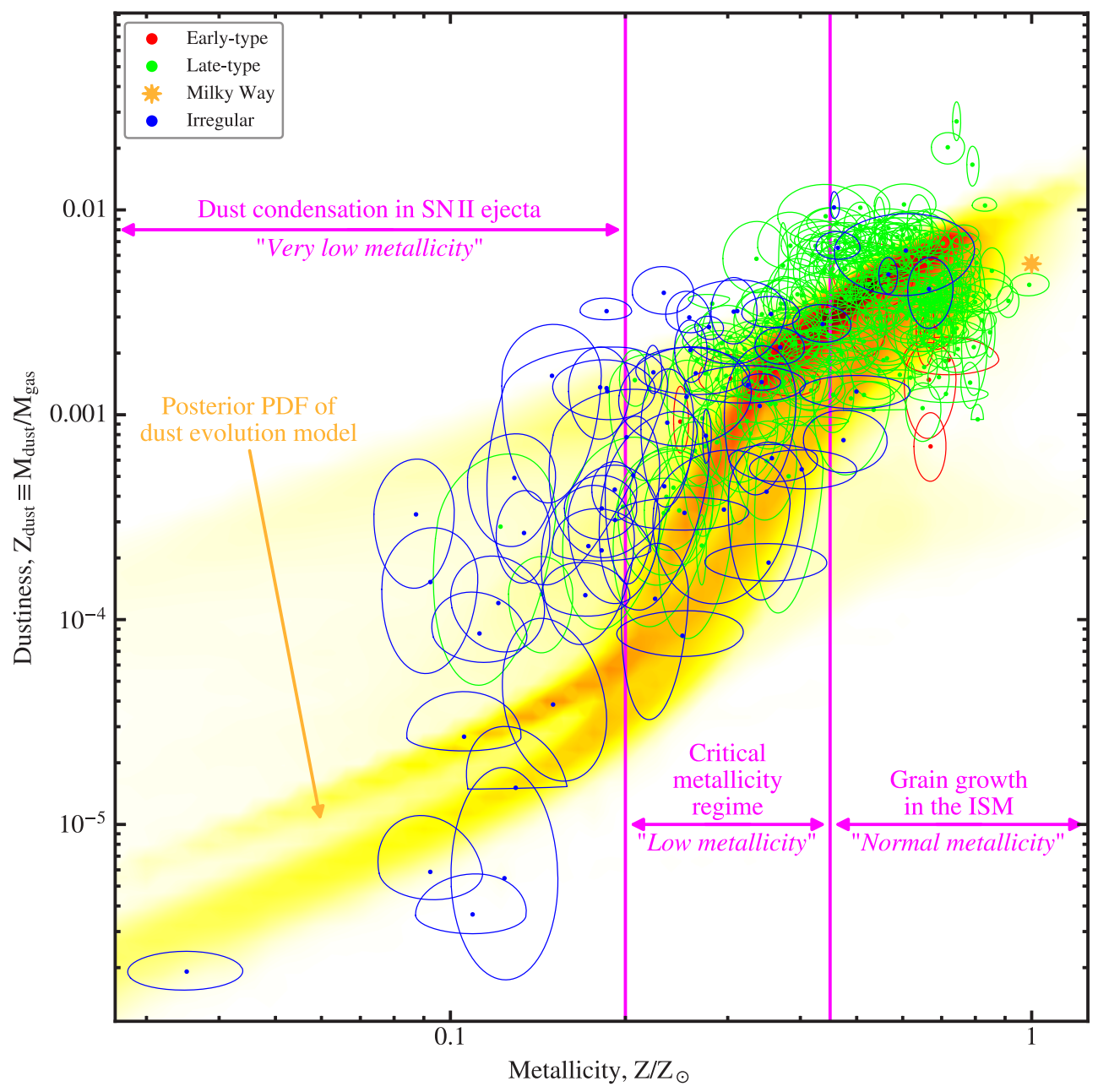

Figure 1. The Skewed Uncertainty Ellipses (SUEs) represent the observed dustiness, as a function of metallicity, of each galaxy in our sample, color-coded according to its type [10]. The yellow-to-red density contours in the background represent the posterior probability density of our dust evolution model, inferred from the observations of galaxies (i.e. the SUEs). We have highlighted the three main dust production regimes in magenta.

We have performed infrared SED modeling of the observed photometry, using the hierachical Bayesian code HerBIE [11]. This model provides a rigorous account of the different sources of uncertainties and is efficient at recovering the intrinsic scatter and correlations of 


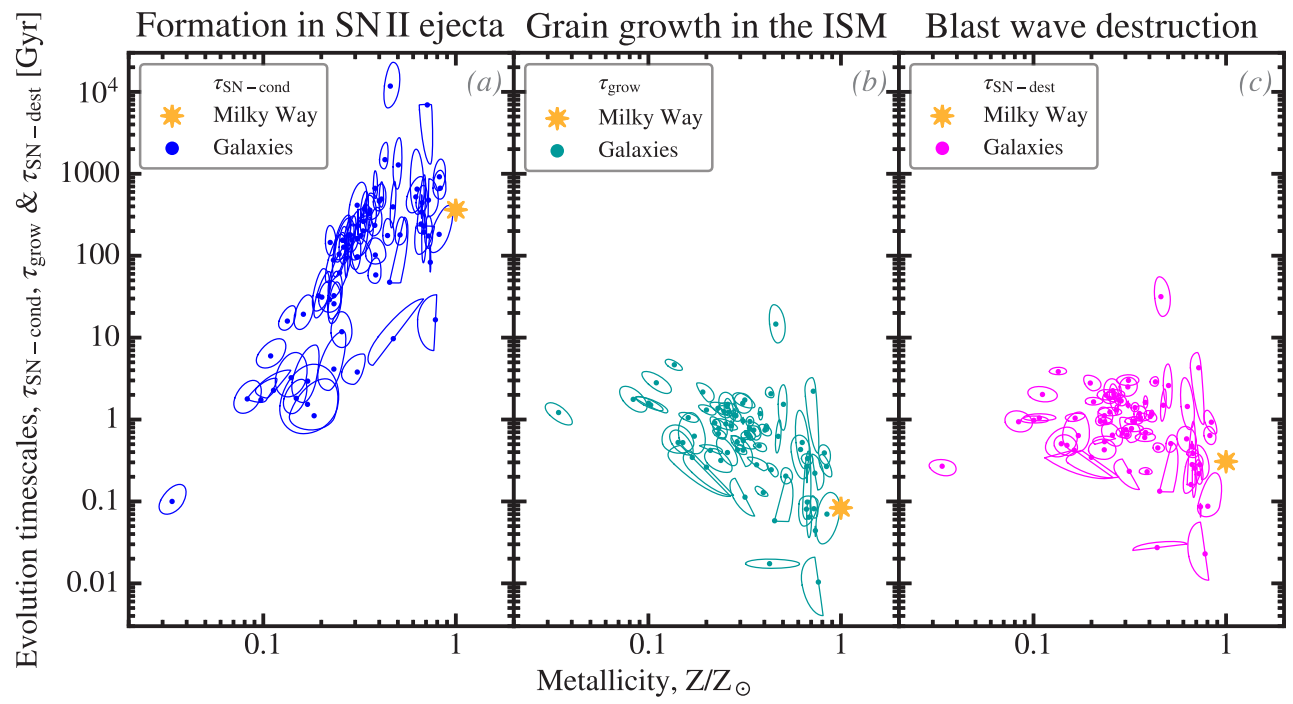

Figure 2. In each panel, the SUEs represent the inferred dust evolution timescales of the galaxies for which all constraints are available, as a function of metallicity [10]. Panel $(a)$ shows the timescale of dust condensation in SN II ejecta, panel (b), the timescale of grain growth in the ISM, and panel $(c)$, the timescale of dust destruction by SN II blast waves.

the studied parameters. We have used the properties of the THEMIS dust model [12]. The results are the Skewed Uncertainty Ellipses (SUEs) in figure 1 [10]. We have displayed the relation between the metallicity and the dustiness (i.e. the dust-to-gas mass ratio).

\section{Constraining dust evolution timescales}

The estimated $M_{\text {dust }}, M_{\text {gas }}, M_{\star}, Z$ and SFR of each galaxy are then used to constrain the parameters of our cosmic dust evolution model. This model, for a given Star Formation History (SFH), computes the evolution, as a function of time, of the masses of stars, gas, heavy elements and dust. At each time step, new stars are formed, depleting the gas. At the end of their lifetime (which depends on their initial mass), these stars return to the ISM a fraction of their gas, newly-formed elements, and dust seeds. The three dust evolution processes we account for (on top of astration, which is straightforward) are: the condensation of grain seeds in SN II ejecta, grain growth in the ISM and dust destruction by SN II blast waves. The efficiency of these different processes are vigorously debated in the literature and they can vary by more than one order of magnitude, depending on the study.

We fit our dust evolution models to our observed estimates in a hierarchical Bayesian way, assuming that each galaxy has a particular SFH (parameterized by its timescale, amplitude, age, infall and outlfow rates), but that the dust evolution efficiencies are universal (i.e. they have the same value for each galaxy). The posterior probability distribution of our dust evolution model is represented as contours in figure 1.

From our inferred dust evolution efficiencies, we can derive dust evolution timescales. These are shown in figure 2. Although we only have one estimate of the three efficiencies for our whole sample, each galaxy has its own set of evolution timescales, because these depend also on the SFR, $M_{\text {gas }}, Z$, etc. The most important conclusions from these trends are the following. 
At very low metallicity, the timescale for dust condensation in SN II is shorter than that of grain growth in the ISM. This is because grain growth is inefficient when the abundance of heavy elements in the gas is low. Grains in these young systems are thus mainly stardust. SN II production is rather inefficient. This is the reason why, in figure 1, very low-metallicity galaxies, lie on the low-dustiness branch of the trend.

At low metallicity, there is a transition between two regimes. The dustiness rises steeply as grain growth in the ISM becomes rapidly more efficient. We are around what [13] have called the critical metallicity. Now, there are enough heavy element atoms in the gas to accrete onto grain surfaces.

At normal metallicity, the dust production is dominated by grain growth in the ISM, with a high efficiency. Although we did not use the constraints provided by the Milky Way, our timescales are consistent with the Galactic ones.

This study also provides methodological insights. First, it demonstrates the instrumental role of dwarf galaxies in understanding dust evolution. These objects indeed sample a metallicity range characterized by a dust production regime different from Solar-metallicity systems. The constraint on the low efficiency of dust production by SN II $\left(\gtrsim 0.03 M_{\odot} / \mathrm{SN}\right.$ in our article) relies entirely on dwarfs. Second, our work is the first rigorous fit of individual dust evolution models to actual observations of galaxies. Most studies before us were simply overlaying dust evolution tracks on top of observed scaling relations. Yet, such a visual comparison is inconsistent in many cases, as it permits interpreting two different quantities of a galaxy with two different ages. Lastly, our results emphasize that grain growth is fast, provided the metallicity is high enough $\left(Z \gtrsim 0.4 Z_{\odot}\right)$. It thus provides a natural mechanism to explain the existence of dusty objects at very high redshifts $(z \gtrsim 6)$, only a few 100 Myrs after reionization.

\section{Prospective: The potential of NIKA2}

The dust evolution study we have just presented was limited because we analyzed galaxies as a whole. The dustiness we estimated for each galaxy was representative of their average conditions, but we know that local grain evolution, at the scale of molecular clouds, is important. Spatially-resolved studies are thus the necessary next step to constrain local dust evolution, that will provide empirical constraints on the effects of gas density and radiation field intensity on the grain properties.

NIKA2 is an instrument providing unprecedentedly deep images of nearby galaxies, at 1.2 and $2 \mathrm{~mm}$, with angular resolutions $12^{\prime \prime}$ and $18^{\prime \prime}$, respectively. It provides unique constraints on the grain properties at spatial scales of a few hundreds of parsecs, close to the size of giant molecular complexes. Figure 3 represents the typical SED of a nearby star-forming galaxy. We see that NIKA2 samples an important spectral domain, where the following physical processes can be probed.

Thermal dust emission, at long-wavelengths, is poorly constrained. Yet, it is crucial to properly estimate dust masses, as it is dominated by cold, massive regions. We have represented the potential presence of a "submm excess", which is likely the result of the inaccuracy of contemporary dust models ( $c f$. [1] for a review). The detailed characterization of dust emission will likely account for this excess. NIKA2 might be instrumental in addressing the origin of this enigmatic effect.

The Anomalous Microwave Emission (AME) is the cm emission shown in red. It originates in fastly spinning small grains [14]. The exact nature of the carriers of this process is still 


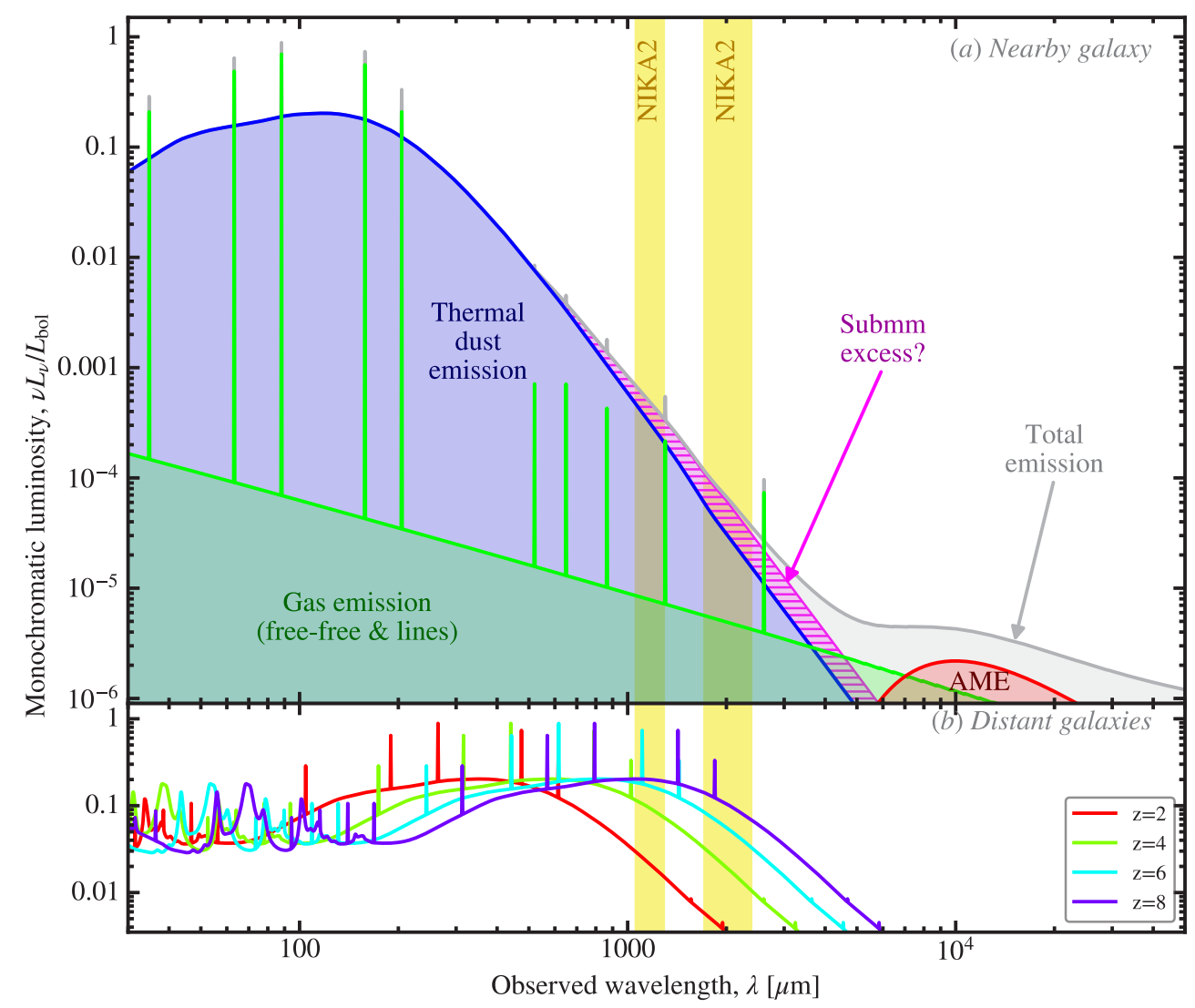

Figure 3. We display the physical processes probed by NIKA2, in the nearby Universe (panel $a$ ), and at high redshift (panel $b$ ). We have represented the tpyical SED of a star-forming galaxy [1].

debated. NIKA2 observations provide the data to properly constrain the baseline required to quantify the AME. NIKA2 data will thus open the possibility to make maps of AME intensity in external galaxies and understand how it correlates with other dust properties, which should give us clues about the nature of its carriers.

The consortium of the NIKA2 guaranteed program, IMEGIN, is currently tackling these challenges ( $c f$. [15] and [16] in this volume).

\section{References}

[1] F. Galliano, M. Galametz, A.P. Jones, ARA\&A 56, 673 (2018)

[2] J.I. Davies, M. Baes, S. Bianchi, A. Jones, S. Madden, M. Xilouris, M. Bocchio, V. Casasola, L. Cassara, C. Clark et al., PASP 129, 044102 (2017), 1609. 06138

[3] C.J.R. Clark, S. Verstocken, S. Bianchi, J. Fritz, S. Viaene, M.W.L. Smith, M. Baes, V. Casasola, L.P. Cassara, J.I. Davies et al., A\&A 609, A37 (2018), 1708.05335

[4] A. Nersesian, E.M. Xilouris, S. Bianchi, F. Galliano, A.P. Jones, M. Baes, V. Casasola, L.P. Cassarà, C.J.R. Clark, J.I. Davies et al., A\&A 624, A80 (2019), 1903.05933 
[5] P. De Vis, A. Jones, S. Viaene, V. Casasola, C.J.R. Clark, M. Baes, S. Bianchi, L.P. Cassara, J.I. Davies, I. De Looze et al., A\&A 623, A5 (2019), 1901.09040

[6] S.C. Madden, A. Rémy-Ruyer, M. Galametz, D. Cormier, V. Lebouteiller, F. Galliano, S. Hony, G.J. Bendo, M.W.L. Smith, M. Pohlen et al., PASP 125, 600 (2013), 1305.2628

[7] A. Rémy-Ruyer, S.C. Madden, F. Galliano, V. Lebouteiller, M. Baes, G.J. Bendo, A. Boselli, L. Ciesla, D. Cormier, A. Cooray et al., A\&A 582, A121 (2015), 1507.05432

[8] A. Rémy-Ruyer, S.C. Madden, F. Galliano, S. Hony, M. Sauvage, G.J. Bendo, H. Roussel, M. Pohlen, M.W.L. Smith, M. Galametz et al., A\&A 557, A95 (2013), 1309. 1371

[9] A. Rémy-Ruyer, S.C. Madden, F. Galliano, M. Galametz, T.T. Takeuchi, R.S. Asano, S. Zhukovska, V. Lebouteiller, D. Cormier, A. Jones et al., A\&A 563, A31 (2014), 1312.3442

[10] F. Galliano, A. Nersesian, S. Bianchi, I. De Looze, S. Roychowdhury, M. Baes, V. Casasola, L.P. Cassará, W. Dobbels, J. Fritz et al., A\&A 649, A18 (2021)

[11] F. Galliano, MNRAS 476, 1445 (2018), 1801.06660

[12] A.P. Jones, M. Köhler, N. Ysard, M. Bocchio, L. Verstraete, A\&A 602, A46 (2017)

[13] R.S. Asano, T.T. Takeuchi, H. Hirashita, A.K. Inoue, Earth, Planets, and Space 65, 213 (2013), 1206.0817

[14] A.C. Bell, T. Onaka, F. Galliano, R. Wu, Y. Doi, H. Kaneda, D. Ishihara, M. Giard, PASJ 71, 123 (2019), 1910.01265

[15] G. Ejlali, R. Adam, P. Ade, H. Ajeddig, P. André, E. , Artis, H. Aussel, A. Beelen, A. Benoît, S. Berta et al., Dust Emission in Galaxies at Millimeter Wavelengths. Cooling of star forming regions in NGC6946, in Proceedings of the International Conference entitled “mm Universe @ NIKA2”, Rome (Italy), June 2021, EPJ Web of conferences, edited by M. De Petris, A. Ferragamo, F. Mayet (2021)

[16] S. Katsioli, R. Adam, P. Ade, H. Ajeddig, P. André, E. , Artis, H. Aussel, A. Beelen, A. Benoît, S. Berta et al., Exploring the millimetre emission in nearby galaxies: analysis of the edge-on galaxy NGC 891, in Proceedings of the International Conference entitled “mm Universe @ NIKA2”, Rome (Italy), June 2021, EPJ Web of conferences, edited by M. De Petris, A. Ferragamo, F. Mayet (2021) 